\title{
Gliosarcoma with Chondrosarcomatous Differentiation: A Case Report
}

\author{
Rajeshwari B ${ }^{1}$, Salapathi Shanmugam ${ }^{1}$, Sadiya Niamath ${ }^{1}$, Mitra Ghosh , Siddartha Ghosh ${ }^{2}$ \\ 'Department of Histopathology, Apollo Speciality Hospitals, Vanagaram, Chennai -600095. India \\ ${ }^{2}$ Department of Neurosciences, Apollo Speciality Hospitals, Vanagaram, Chennai -600095. India
}

\begin{abstract}
Gliosarcoma (GS) is a variant of IDH wild type of glioblastoma (WHO grade IV). It is characterized by biphasic tissue pattern showing glial and mesenchymal components. The glial component is usually similar to glioblastoma and the sarcomatous component is usually spindle cell type. Rarely the sarcomatous component can show additional lines of mesenchymal differentiation like cartilage, bone, smooth muscle, skeletal muscles etc. These tumours are more firm and discrete because of the high content of reticulin and collagen in the sarcomatous component. Originally these tumours were thought to arise from sarcomatous transformation of proliferating blood vessels or dedifferentiation of an advanced glioma. The recent studies show that both glial and sarcomatous component show identical mutations in p53, PTEN and TERT genes and suggest a monoclonal origin from a common stem cell. There is no significant difference in prognosis between glioblastoma and gliosarcoma. Here we report a case of gliosarcoma with chondrosarcomatous differentiation in a 52 year old gentleman. The case is presented here for its rarity.
\end{abstract}

Keywords: Chondrosarcoma, Glioblastoma, Gliosarcoma, PTEN, TERT

\section{Introduction}

Gliosarcoma (GS) is a variant of IDH-wild type glioblastoma characterized by biphasic tissue pattern with alternating areas of glial and mesenchymal (sarcomatous) differentiation. It is a WHO grade IV neoplasm. ${ }^{[1]}$ These tumours constitute approximately $2 \%$ of all malignant glial neoplasms with a similar age and gender distribution as glioblastomas. ${ }^{[2]}$ They commonly affect adults in the fifth to sixth decades of life with a male:female ratio of 1.4:1 to $1.8: 1 .^{[3]}$ They occurs commonly in the cerebral hemispheres. ${ }^{[1]}$ The sarcomatous areas commonly resemble a fibrosarcoma, but may also show a variety of lines of mesenchymal differentiation including bone, cartilage, or muscle. ${ }^{[1,3]}$ The demonstration of a clearly malignant mesenchymal GFAP negative component is important to distinguish true gliosarcoma from glioblastoma with florid fibroblastic proliferation. ${ }^{[1]}$ Presence of malignant chondroid elements is rare in Gliosarcoma. Here we report a case of gliosarcoma with chondrosarcomatous differentiation.

\section{Case Report}

52 year old gentleman presented with progressive generalized weakness, lethargy and slowness of movements for a duration of 6 months. There was history of recent memory loss and change in the behaviour. MRI showed well defined heterogeneously enhancing mass lesion seen in proximal body of corpus callosum and extending into bilateral frontal lobes with small cystic and hemorrhagic areas (Figure 1). There was mass effect on frontal horn of both lateral ventricles and perilesional edema in bilateral frontal lobes. MRI features were suggestive of high grade glioma. Intraoperatively the lesion appeared grayish and vascular with variegated consistency and calcified areas. The lesion was seen involving the anterior and middle part of corpus callosum. Intra operatively the specimen was sent for frozen section. The squash smears showed features of a high grade glioma. Tumour was excised and sent for histopathological examination. The formalin fixed paraffin embedded sections showed a biphasic neoplasm comprising of malignant glial and mesenchymal components. The glial component showed increased cellularity with cells arranged in diffuse infiltrating sheets, having pleomorphic hyperchromatic nuclei arranged in fibrillary matrix. Many cells having bizarre nuclear atypia with bi and multinucleation were seen. Interspersed gemistocytic cells were also noted. Mitoses were increased. Areas of geographic and pseudopalisading necrosis with microvascular proliferation were noted. Mesenchymal component showed spindle cells arranged in fascicles and sheets, with nuclear atypia and mitoses with a collagenised stroma. Areas of well differentiated cartilagenous elements comprising of atypical chondrocytes having bizarre oval to spindle nuclei lying in lacunae, surrounded by chondroid stroma were seen. Few mitoses were noted in these chondroid areas. Masson's trichrome and reticulin stains showed increased deposition of collagen within the mesenchymal areas (Figure 2 \& Figure 3). On immunohistochemistry, the glial component was GFAP positive. The mesenchymal component was positive for 
vimentin and was GFAP negative (Figure 4). IDH1 was negative in both glial and mesenchymal components. p53 was positive in both glial and sarcomatous areas. S100 was focally positive in chondroid areas(Figure 4). PTEN was positive in low grade areas and showed loss of expression in high grade areas near the chondroid areas. The case was reported as gliosarcoma with chondrosarcomatous differentiation.

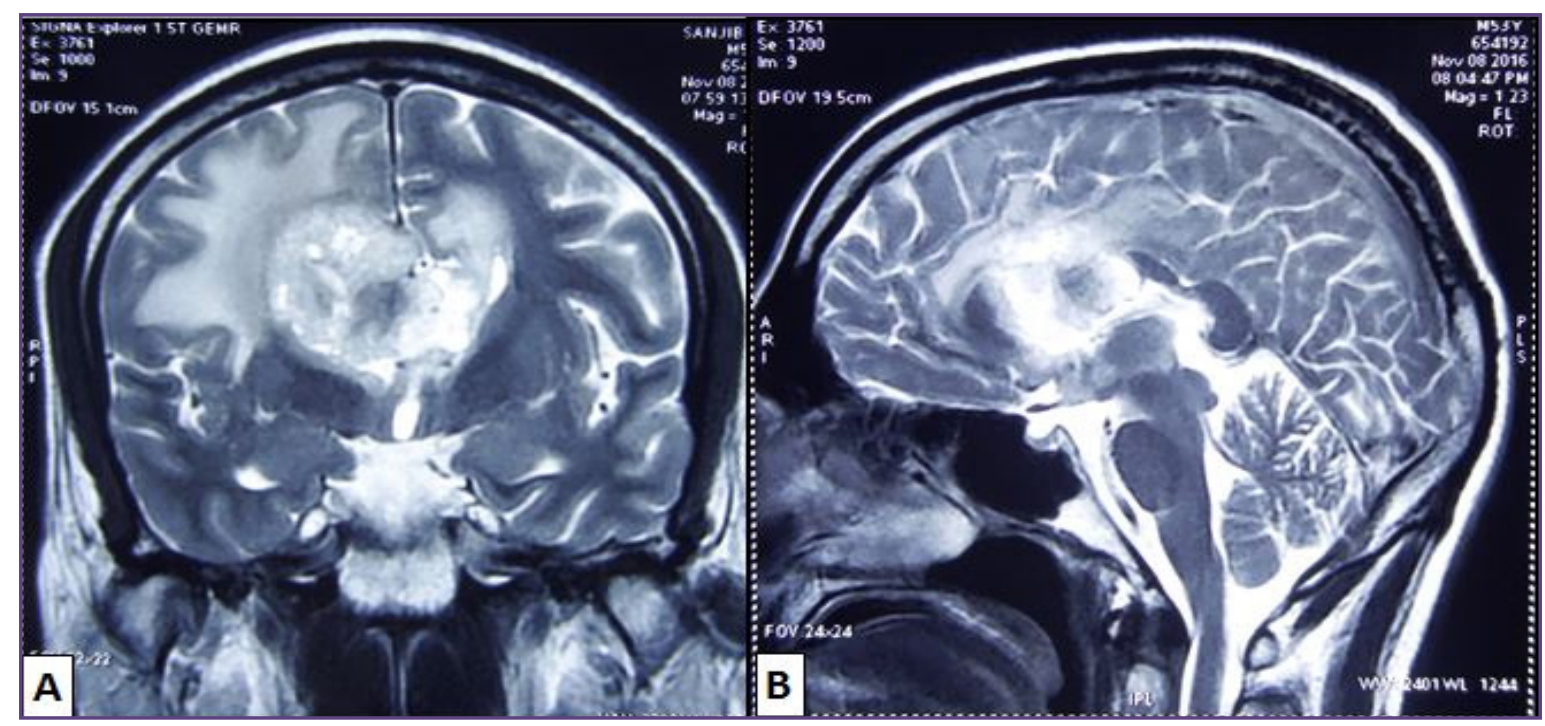

Fig. 1: A \& B: MRI brain showing heterogeneously enhancing mass lesion seen in proximal body of corpus callosum and extending into bilateral frontal lobe with small cystic and hemorrhagic areas with perilesional edema, suggestive of high grade glioma.

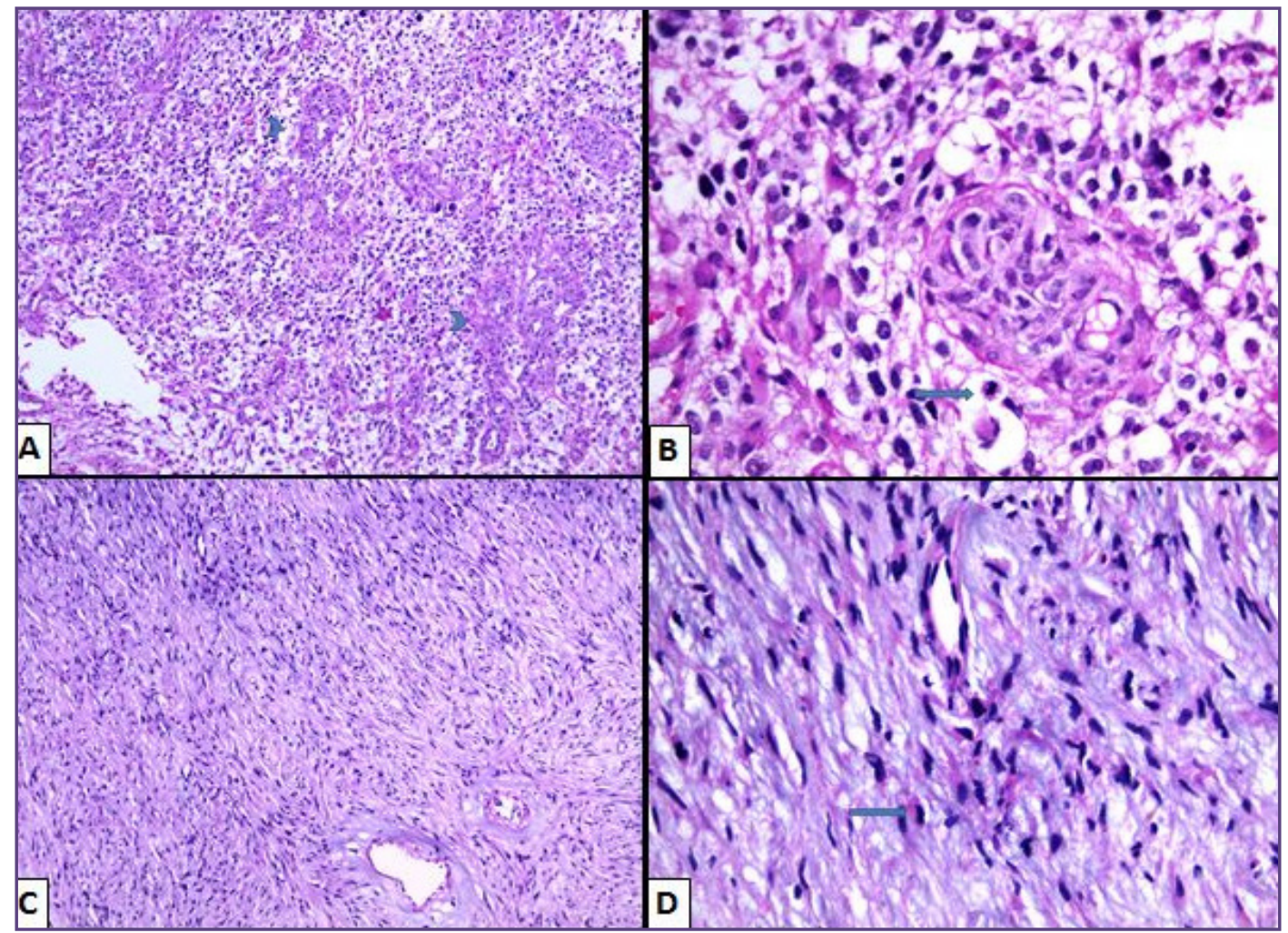

Fig. 2: A \&B: High grade glial component showing vascular endothelial proliferation(arrow heads) and mitoses(arrows) C \& D: Mesenchymal component showing atypical spindle cells in a collagenised stroma with mitoses (arrow), H \& E stain, 100x \& 400x. 


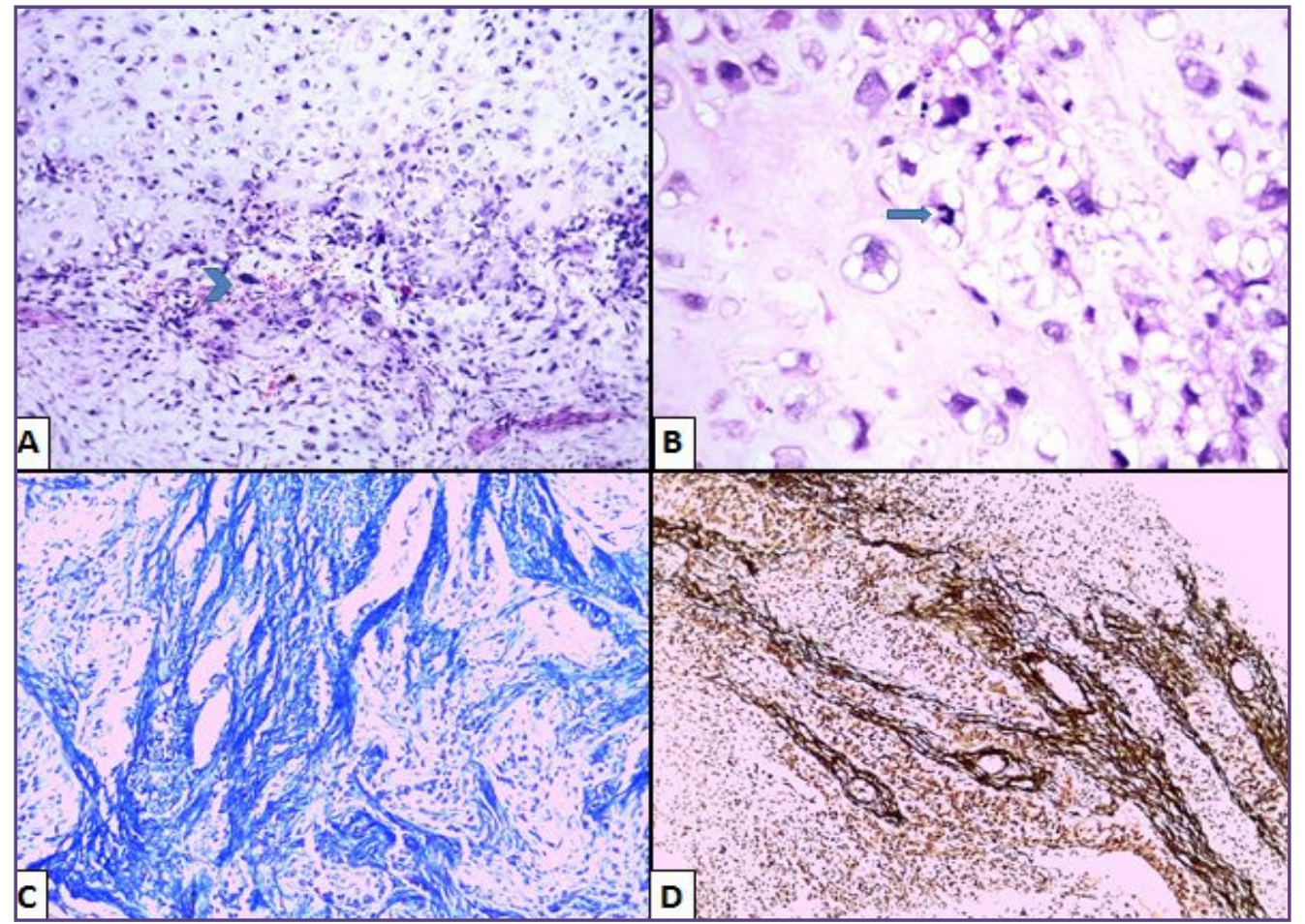

Fig. 3: A \& B: Atypical cartilagenous areas in mesenchymal component showing bizarre chondrocytes (arrow head) and mitosis (arrow). H \& E stain, 100x \& 400x C \& D: Masson's trichrome and Reticulin stains to demonstrate increased collagen in the mesenchymal areas. 100x.

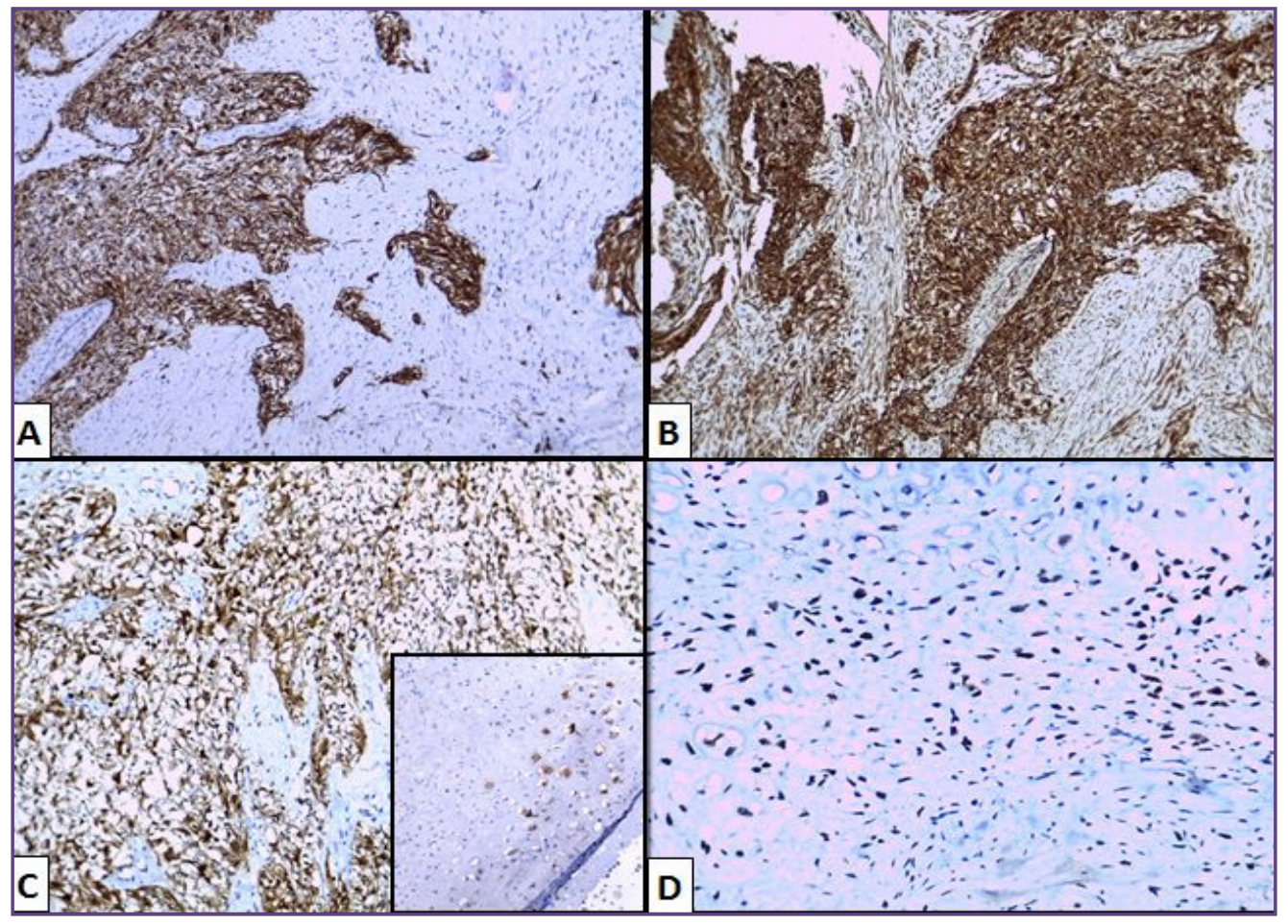

Fig. 4: Immunohistochemistry A : Tumour cells positive for GFAP B: Vimentin positive in GFAP negative mesenchymal component. C: S100 is positive in tumour cells and atypical chondrocytes (Inset). D: p53 is positive in both glial and sarcomatous areas. 100x. 


\section{Discussion}

Gliosarcomas were first described in 1895 by Heinrich Stroebe ${ }^{[4]}$ In 1955, Feigin et al. defined gliosarcomas as glioblastoma multiforme that acquired the features of a sarcoma from the proliferating vessels . Hence, gliosarcoma is sometimes referred to as "Feigin's tumor". ${ }^{[5]}$ Radiologically, presence of high sarcomatous component gives the appearance of a well circumscribed hyperdense mass with homogenous contrast enhancement and hence may mimic meningioma. ${ }^{[1]}$ Cases with additional lines of mesenchymal differentiation, such as the formation of cartilage, osteoid- chondroid tissue, smooth and striated muscle, and even lipomatous features have also been reported. Primitive neuronal components occur rarely. ${ }^{[1]}$ In some reports, it was demonstrated that neoplastic astrocytes are capable of differentiating into cartilage through mucopolysaccharide deposition, which can evolve to form a chondroid matrix. ${ }^{[6]}$ Our case showed biphasic pattern with a high grade astrocytic and sarcomatous components. The sarcomatous component showed chondrosarcomatous differentiation. The features were morphologically and immunohistochemically consistent with gliosarcoma showing chondrosarcomatous differentiation.

Early reports have suggested that the sarcomatous components originated from the neoplastic transformation of the hyperplastic blood vessels which are commonly found in high-grade gliomas ${ }^{[3]}$ Another hypothesis was that sarcomatous component resulted from dedifferentiation of an advanced glioma with subsequent loss of GFAP expression and acquisition of a sarcomatous phenotype. The recent cytogenetic studies suggest that both components have monoclonal origin from neoplastic glial cells with presence of identical mutations in TP53, PTEN and TERT mutations in glial and sarcomatous components. According to this finding, it is most likely that sarcomatous and gliomatous cells are derived from a common stem cell. ${ }^{[1,7,8]}$ Numerous genetic studies of gliosarcomas support this monoclonal hypothesis. ${ }^{[2]}$ There is no significant prognostic difference between gliosarcoma and typical glioblastoma since the sarcomatous component is prognostically insignificant. ${ }^{[9,10]}$

\section{Conclusion}

Gliosarcoma is a WHO grade IV neoplasm with a biphasic pattern showing glial and sarcomatous component. The glial component is usually similar to glioblastoma and sarcomatous component is usually of spindle cell type. Different types of mesenchymal differentiation are known to occur and it is rare to find chondrosarcomatous differentiation. There is no significant prognostic difference between glioblastoma and gliosarcoma. The case is presented here for its rarity.

\section{Acknowledgements}

We sincerely acknowledge our technical staff, $\mathrm{Mr} \mathrm{R}$ Bhuvaneshwaran, Mrs P Shivashankari, Mrs Lavanya Latha, Mrs J Rukmani, for their technical help

\section{References}

1. Burger PC, Giangaspero F, Ohgaki H, Biernat W. In: Louis DN, Ohgaki H, Wiestler DO, Cavenee WK editors. WHO Classification of Tumours of the Central Nervous System, Revised 4th edition. Lyon: IARC Press; 2016. p. 48-9.

2. Barut F, Kandemir NO, Ozdamar SO, Gul S, Bektas S, Gun $\mathrm{BD}$ et al. Gliosarcoma with chondroblastic osteosarcomatous differentation: report of two case with clinicopathologic and immunohistochemical features. Turk Neurosurg 2009; 19:417-22.

3. Ravisankar S, Chander RV, Devadoss PK. Pediatric gliosarcoma with fibrosarcomatous differentiation: Report of a rare case. Indian J Pathol Microbiol 2012;55:521-4.

4. Barresi V, Cerasoli S, Morigi F, Cremonini AM, Volpini M, Tuccari G. Gliosarcoma with features of osteoblastic osteosarcoma: a review. Arch Pathol Lab Med 2006;130:1208-11.

5. Feigin IH, Gross SW: Sarcoma arising in glioblastoma of the brain. Am J Pathol 31:633-653,1955.

6. Yao K, Qi, X.-L, Mei, X, Jiang T. Gliosarcoma with primitive neuroectodermal, osseous, cartilage and adipocyte differentiation: a case report. Int J Clin Exp Pathol 2015; 8; 2079-84.

7. Alatakis S, Stuckey S, Siu K, McLean C. Gliosarcoma with osteosarcomatous differentiation: a review of radiological and pathological features. J Clin Neurosci 2004;11:650-6.

8. Machuca TN, Prevedello DM, Pope LZ, Haratz SS, Araujo JC, Torres LF. Gliosarcoma: report of four cases with immunohistochemical findings. Arq Neuropsiquiatr 2004;62:608-12.

9. Brat DJ, Perry A. Astrocytic and Oligodendroglial Tumors. In: Practical Surgical Neuropathology: A Diagnostic approach. Philadelphia: Churchil Livingstone Elsevier;2010. p. 70.

10. Winkler PA, Buttner A, Tomezzoli A, Weis S. Histologically repeatedly confirmed gliosarcoma with long survival: review of the literature and report of a case. Acta Neurochir 2000;142:91- 5 . 